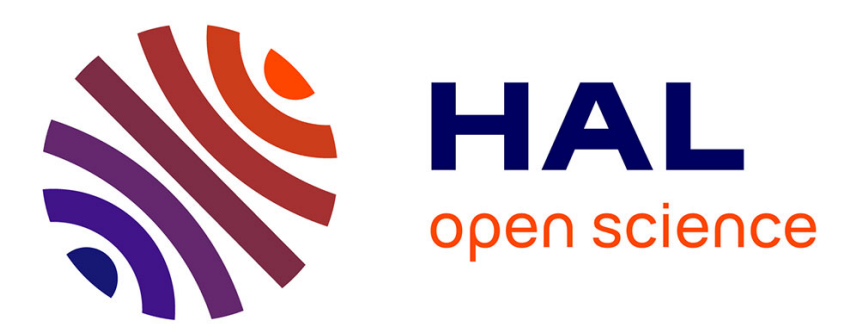

\title{
Homogénéisation des matériaux constituant les condensateurs bobinés à films métallisés
}

Ch. Joubert, G. Rojat, A. Béroual

\section{To cite this version:}

Ch. Joubert, G. Rojat, A. Béroual. Homogénéisation des matériaux constituant les condensateurs bobinés à films métallisés. Journal de Physique III, 1997, 7 (7), pp.1549-1559. 10.1051/jp3:1997207 . jpa-00249665

\section{HAL Id: jpa-00249665 https://hal.science/jpa-00249665}

Submitted on 1 Jan 1997

HAL is a multi-disciplinary open access archive for the deposit and dissemination of scientific research documents, whether they are published or not. The documents may come from teaching and research institutions in France or abroad, or from public or private research centers.
L'archive ouverte pluridisciplinaire HAL, est destinée au dépôt et à la diffusion de documents scientifiques de niveau recherche, publiés ou non, émanant des établissements d'enseignement et de recherche français ou étrangers, des laboratoires publics ou privés. 


\title{
Homogénéisation des matériaux constituant les condensateurs bobinés à films métallisés
}

\author{
Ch. Joubert, G. Rojat et A. Béroual (*)

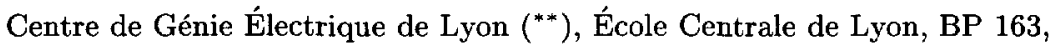 \\ 69131 Ecully Cedex, France
}

(Reçu le 28 janvier 1997, révisé le 28 mars 1997, accepté le $1^{\text {er }}$ avrll 1997)

\author{
PACS.41.20.-q - Electric, magnetic and electromagnetic fields \\ PACS.02.60.- $\mathrm{x}$ - Numerical approximation and analysis \\ PACS.07.55.-w - Magnetic components, instruments and techniques
}

\begin{abstract}
Résumé. - La structure des condensateurs métallisés est complexe et les tailles des éléments constitutifs sont dissemblables. En conséquence, le calcul direct de champs et de densités de courant est impossible. Dans cet article, nous donnons quelques propriétés macroscopiques (impédances, admittances) du bobinage, obtenues grâce à une méthode d'homogénéisation. Ces propriétés permettent de calculer la répartition des courants et des échauffements dans les condensateurs.
\end{abstract}

\begin{abstract}
The structure of metallized capacitors is complex and the sizes of the constituting elements are dissimilar. Consequently, direct computation of fields and current densities is impossible. In this paper, we give some macroscopic properties (Impedances, admittances) of the capacitor winding, obtained thanks to an homogeneization method. These properties are helpful to compute the current and heating distribution in capacitors.
\end{abstract}

\section{Introduction}

Les condensateurs bobinés à films métallisés sont constitués de deux films de polymère recouverts, sur une face, d'une fine couche de métaux (zinc, aluminium) qui constitue ainsi une armature. Pour former le condensateur, les films sont enroulés sur un support isolant. Sur les deux extrémités du bobinage ainsi constitué, on projette un alliage de zinc fondu qui établit un contact avec les armatures (Fig. 1). Ce procédé, appelé procédé de "schoopage", permet la connexion de fils métalliques qui relient le condensateur au circuit extérieur $[1,2]$.

Les constituants des condensateurs à films bobinés métallisés ont des dimensions qui varient dans de larges proportions : le diamètre des schoopages est de l'ordre de $10^{-2} \mathrm{~m}$ alors que leur épaisseur est voisine de $10^{-3} \mathrm{~m}$. Les films de polymère ont une épaisseur proche de $10^{-5} \mathrm{~m}$ pour une longueur de l'ordre de $10^{2} \mathrm{~m}$ correspondant à plusieurs milliers de tours. Enfin, l'épaisseur des couches métalliques ne dépasse pas $10^{-7} \mathrm{~m}$. En conséquence, il est impossible de calculer directement, par des méthodes analytiques ou numériques, la répartition du courant et du champ

(*) Auteur auquel doit être adressée la correspondance (e-mail : beroual@trotek.ec-lyon.fr) $\left({ }^{* *}\right)$ UPRESA CNRS 5005 


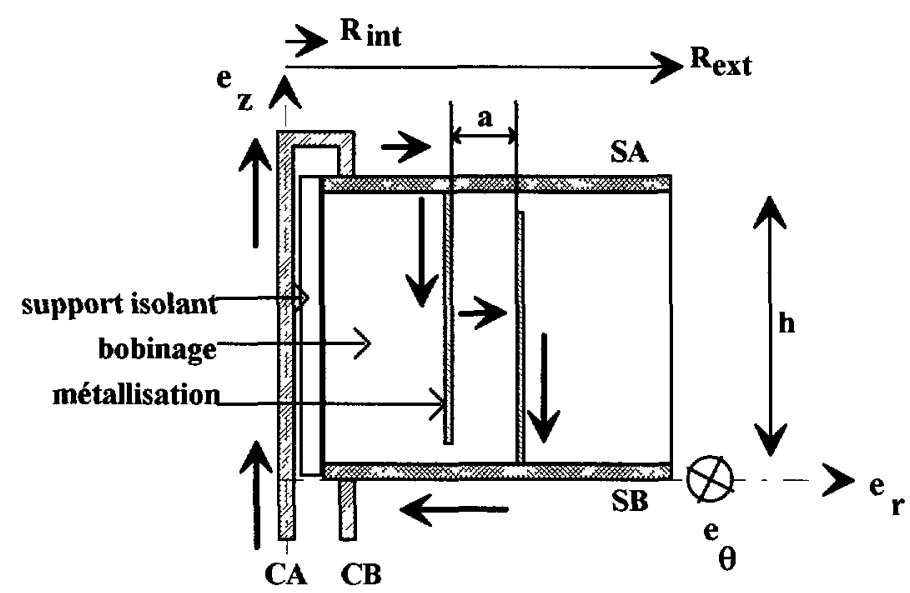

Fig 1. - Structure des condensateurs bobinés à films métallisés.

[Structure of metallized capacitors.]

magnétique à l'intérieur des condensateurs. Connaître la densité de courant dans un condensateur est pourtant nécessaire afin de déterminer les échauffements, d'optimiser la structure du condensateur et de maîtriser ses caractéristiques (impédance, rayonnement émis...).

Pour mener à bien les calculs de champs, il est cependant possible d'utiliser des paramètres macroscopiques homogènes qui décrivent, à l'échelle du millimètre, le comportement des éléments constitutifs du condensateur $[3,4]$. Ces paramètres macroscopiques doivent être obtenus à partir de la structure microscopique de l'objet étudié, c'est-à-dire de la disposition des armatures métalliques, des couches diélectriques et des propriétés du schoopage. Dans cet article, nous nous intéressons ainsi aux impédances et admittances macroscopiques qui caractérisent le condensateur.

Afin de faciliter l'étude, les courants qui traversent ces impédances et admittances sont décomposés en deux grandeurs : les courants capacitifs et les courants induits dans les métallisations. Nous supposons aussi, dans un soucis de simplification, qu'il n'existe pas de couplage résistif ou capacitif entre ces deux types de courants mais qu'il existe un couplage magnétique. Cette hypothèse sur le couplage des courants est validée expérimentalement.

\section{Courants capacitifs et courants dans le schoopage}

Le courant parcourt le condensateur selon le chemın suivant (Fig. 1) . 1l arrive par le conducteur $\mathrm{CA}$, circule dans le schoopage SA suivant la direction $\mathbf{e}_{\mathbf{r}}$, passe dans la métallisation d'un film, traverse le diélectrique sous forme de courant de déplacement, rejoint la métallisation du deuxième film puis le schoopage SB et revient enfin par le conducteur CB.

Nous appelons courant capacitif le courant, de densité surfacique $\underline{J}_{\mathrm{sc}}$, qui circule suivant $\mathbf{e}_{z}$. Ce courant dépend de la circulation du champ électrique $\underline{\zeta}$ entre les schoopages:

$$
\underline{J}_{\mathrm{sc}}=\underline{\zeta}_{\mathrm{Sc}}
$$

avec :

$$
\underline{\zeta}=\underline{V}-j \omega \underline{A} h
$$




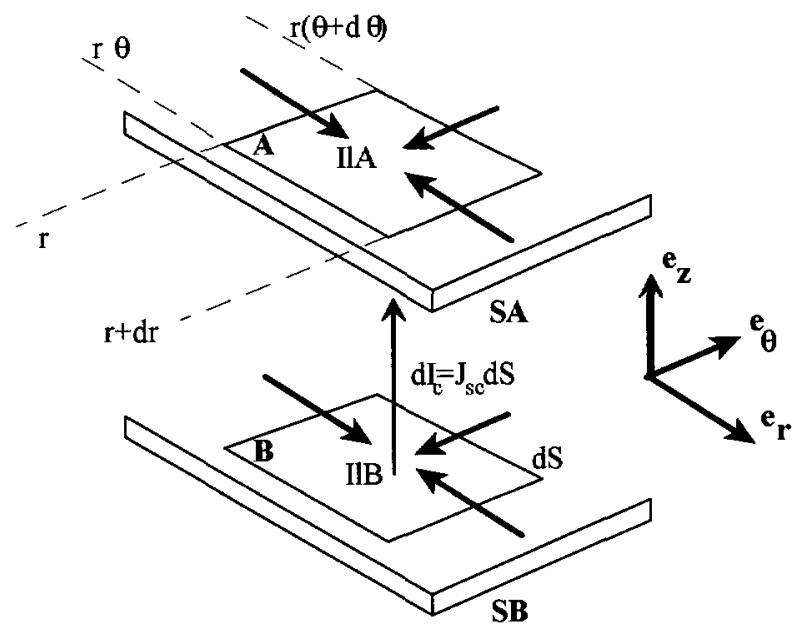

Fig. 2. - Courant dans le bobinage et courants dans les schoopages.

[Current in the winding and currents in the schooping.]

où $\underline{V}, \underline{A}$ et $\underline{Y}_{\mathrm{sc}}$ représentent respectivement la différence de potentiel électrique entre les schoopages, la composante axiale du potentiel vecteur magnétique et l'admittance surfacique entre les deux schoopages. Cette admittance surfacique peut être exprimée par :

$$
\underline{Y}_{\mathrm{sc}}=\frac{j C_{\mathrm{s}} \omega}{1+j \frac{C_{\mathrm{s}}}{G_{\mathrm{s}}} \omega}
$$

où $C_{\mathrm{s}}$ et $G_{\mathrm{s}}$ représentent des paramètres qui dépendent de la résistance $R_{\square}$ de la métallisation, des caractéristiques des films de polymère ainsi que de la fréquence des courants [5]. Une valeur approchée de $C_{\mathrm{s}}$ peut être obtenue en divisant la capacité du condensateur par la surface d'un schoopage. Pour obtenir le paramètre $G_{\mathrm{s}}$, homogène à une conductance par unité de surface, il est nécessaire d'étudier la répartition du champ électrique le long des armatures. La méthode employée a été développée par ailleurs [6].

Le courant qui circule suivant $\mathbf{e}_{\mathrm{r}}$, de densité linéique $\underline{I}_{1}$, dépend, pour sa part, des caractéristiques du schoopage par l'intermédiaire d'une impédance linéique $\underline{z}_{\mathrm{s}}$. Si la fréquence des courants est telle que l'épaisseur de peau $\delta$ dans le métal est très petite devant l'épaisseur du schoopage, l'impédance $\underline{z}_{\mathrm{s}}$ s'écrit :

$$
\underline{z}_{\mathrm{s}}=\frac{(1+j) \rho_{\mathrm{s}}}{\delta}
$$

où $\rho_{\mathrm{s}}$ représente la résistivité du schoopage.

Dans le schoopage A (Fig. 2), les composantes du courant suivant les axes $\mathbf{e}_{\mathbf{r}}$ et $\mathbf{e}_{\theta}$ s'écrivent :

$$
\begin{aligned}
\underline{I}_{1 \mathrm{Ar}} r \mathrm{~d} \theta & =-\frac{1}{\underline{z}_{\mathrm{s}}} \frac{r \mathrm{~d} \theta}{\mathrm{d} r}\left[\underline{V}_{\mathrm{A}}(r, \theta)-\underline{V}_{\mathrm{A}}(r+\mathrm{d} r, \theta)\right] \\
\underline{I}_{1 \mathrm{~A} \theta} \mathrm{d} r & =-\frac{1}{\underline{z}_{\mathrm{s}}} \frac{\mathrm{d} r}{r \mathrm{~d} \theta}\left[\underline{V}_{\mathrm{A}}(r, \theta)-\underline{V}_{\mathrm{A}}(r, \theta+\mathrm{d} \theta)\right]
\end{aligned}
$$

où $\underline{V}_{\mathrm{A}}(r, \theta)$ représente le potentiel électrique du schoopage $\mathrm{A}$ au point de coordonnées $(r, \theta)$. 
En prenant $\mathrm{d} r$ et $\mathrm{d} \theta$ suffisamment petits, l'équation (5) devient :

$$
\begin{aligned}
& \underline{I}_{1 \mathrm{Ar}}=-\frac{1}{\underline{z}_{\mathrm{s}}} \frac{\partial \underline{V}_{\mathrm{A}}(r, \theta)}{\partial r} \\
& \underline{I}_{1 \mathrm{~A} \theta}=-\frac{1}{z_{\mathrm{s}}} \frac{1}{r} \frac{\partial \underline{V}_{\mathrm{A}}(r, \theta)}{\partial \theta}
\end{aligned}
$$

soit :

$$
\underline{\mathbf{I}}_{1 \mathrm{~A}}=-\frac{1}{\underline{z}_{\mathrm{s}}} \operatorname{grad} \underline{V}_{\mathrm{A}} .
$$

On obtient de même, pour le schoopage B :

$$
\underline{\mathbf{I}}_{1 \mathrm{~B}}=-\frac{1}{\underline{z}_{\mathrm{s}}} \operatorname{grad} \underline{V}_{\mathrm{B}}
$$

où $\underline{V}_{\mathrm{B}}(r, \theta)$ et $\underline{\mathrm{I}}_{1 \mathrm{~B}}$ représentent respectivement la répartition de potentiel électrique et la densité linéique de courant dans le schoopage $\mathrm{B}$.

Enfin, en remarquant que $\underline{V}=\underline{V}_{\mathrm{B}}-\underline{V}_{\mathrm{A}}$ et en posant $\underline{\mathbf{I}}_{1}=\underline{\mathbf{I}}_{1 \mathrm{~B}}-\underline{\mathbf{I}}_{1 \mathrm{~A}}$, il vient :

$$
\underline{I}_{1}=-\frac{1}{z_{s}} \operatorname{grad} \underline{V} \text {. }
$$

Quand le conducteur d'alimentation du condensateur est placé sur l'axe $\mathbf{e}_{\mathrm{r}}$ (Fig. 1), il apparaît une symétrie axiale dans la répartition des champs et des courants. Cette symétrie est conservée quand le conducteur d'alimentation est un cylindre placé à l'extérieur du condensateur et concentrique à ce dernier (conducteur de retour "en cloche"). Cette disposition permet de réduire l'inductance série équivalente du condensateur et donc d'augmenter sa plage d'utilisation en fréquence $[7,8]$.

Il n'est toutefois pas toujours envisageable de conserver la symétrie du dispositif. Ainsi, dans les utilisations usuelles des condensateurs, le conducteur d'alimentation est souvent placé à l'extérieur de façon non symétrique (Fig. 3a).

\section{Courants induits dans les métallisations}

La présence d'un conducteur de retour du courant à proximité du bobinage d'un condensateur entraîne l'apparition d'un champ magnétique qui traverse les métallisations du condensateur (Fig. 3a) [9]. Ce champ magnétique produit à son tour des courants induits de densité surfacique $I_{s m}$ qui circulent dans les métallisations des condensateurs comme indiqué sur la figure $3 \mathrm{~b}$. Le trajet de ces courants peut être décomposé grossièrement en quatre segments : deux segments $\mathrm{AB}$ et $\mathrm{A}$ 'B' parallèles à l'axe de symétrie de révolution du condensateur ; un segment AA' qui suit le bord de la métallisation ; enfin un segment BB' compris dans le schoopage.

3.1. RÉPARTITION DES COURANTS INDUits. - Afin de préciser la répartition des courants induits, nous supposons que le bobinage du condensateur est plongé dans une induction magnétique homogène d'intensité $B_{0}$. La portion de métallisation de hauteur $h$ et de longueur $\pi R_{\mathrm{m}}$ correspondant à un demi-tour de bobinage pour un rayon moyen $R_{\mathrm{m}}=\frac{1}{2}\left(R_{\mathrm{nt}}+R_{\mathrm{ext}}\right)$ est représentée sur la figure $3 \mathrm{c}$. Cette portion est soumise à une induction magnétique normale :

$$
B\left(R_{\mathrm{m}} \theta, z\right)=B_{0} \cos \left(\frac{\pi}{\pi R_{\mathrm{m}}} R_{\mathrm{m}} \theta\right) .
$$




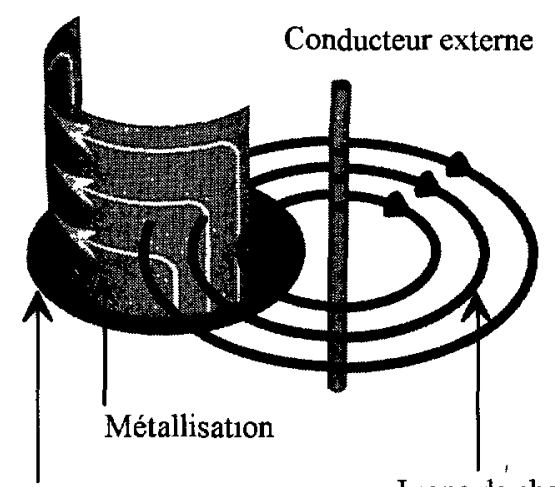

a) Schoopage

Ligne de champ magnétique

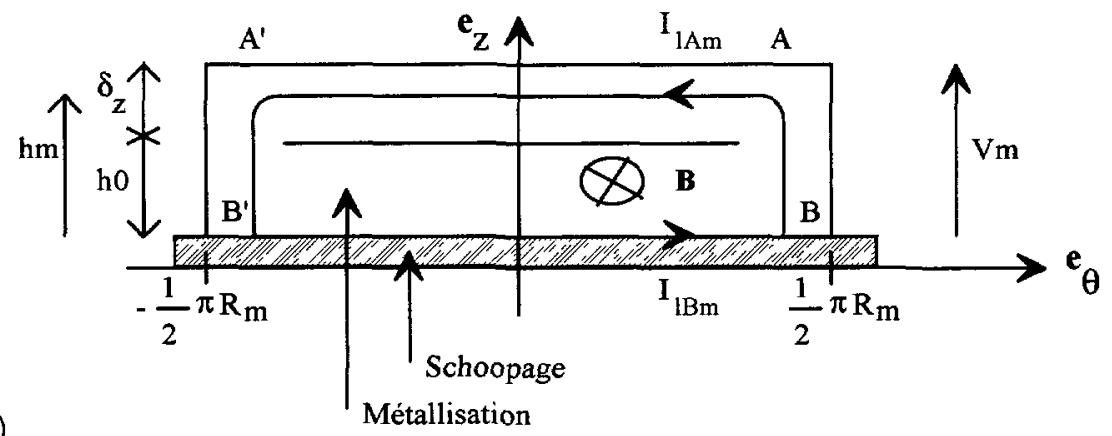

b)

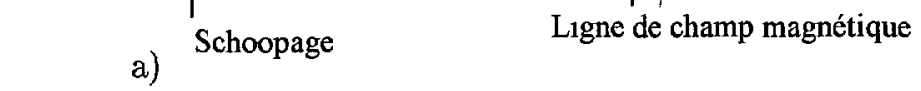

Conducteur externe

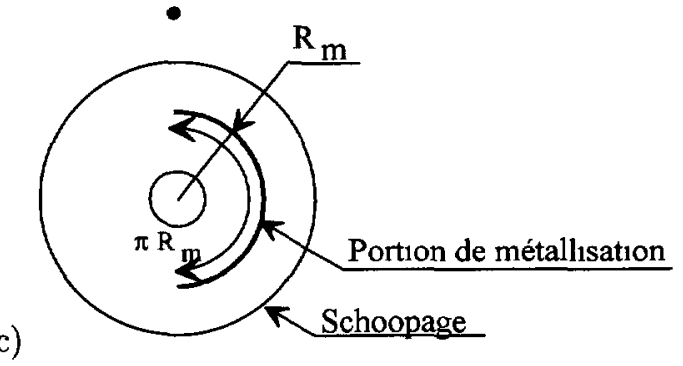

Fig. 3. - Courants induits. a) Perspective ; b) vue développée de la métallisation, c) vue de dessus. [Eddy currents. a) Perspectıve view; b) developed diagram of the metallization; c) view from above.]

La répartition de champ électrique dans la métallisation obéit à la relation :

$$
\operatorname{rot} \mathbf{E}=-j \omega \mathbf{B}
$$

soit :

$$
\Delta \mathbf{E}=\jmath \omega \operatorname{rot} \mathbf{B}
$$

avec :

$$
\operatorname{rot} \mathbf{B}=\frac{B_{0}}{R_{\mathrm{m}}} \cos (\theta) \mathbf{e}_{z} .
$$


La composante du champ électrique parallèle à l'axe des abscisses obéit à l'équation :

$$
\Delta E_{\theta}=0 .
$$

En tenant compte des conditions aux limites:

$$
\begin{aligned}
E_{\theta}\left(-\frac{1}{2} \pi R_{\mathrm{m}}, z\right) & =E_{\theta}\left(\frac{1}{2} \pi R_{\mathrm{m}}, z\right)=0 \\
E_{\theta}(0, z) & =-E_{\theta}(0,-z)
\end{aligned}
$$

la composante horizontale du champ électrique est alors de la forme :

$$
E_{\theta}=E_{0 \theta} \operatorname{sh}\left(\frac{\pi}{\pi R_{\mathrm{m}}} z\right) \cos (\theta) .
$$

Cette composante varie donc de façon continue de sa valeur maximale $E_{x}(0, h)$ (au bord de la métallisation) à zéro (sur le schoopage). Pour faciliter les calculs, nous supposons cependant que le courant se distribue de façon uniforme dans une zone de largeur $\delta_{z}$ au bord de l'armature, $\delta_{z}$ étant défini par :

$$
\frac{E_{x}(0, h)}{E_{x}\left(0, h-\delta_{x}\right)}=e
$$

soit, en posant $\lambda=\frac{2 h}{R_{\mathrm{int}}+R_{\mathrm{ext}}}$ :

$$
\delta_{z}=\left[1-\frac{1}{\lambda} \operatorname{argsh}\left(\frac{1}{e} \operatorname{sh}(\lambda)\right)\right] h .
$$

L'évolution du paramètre $\alpha=\delta_{z} / h$ en fonction de $\lambda$ est représentée sur la figure 4 . On peut remarquer que, pour les dimensions usuelles de condensateurs, $\delta_{z}$ est du même ordre de grandeur que la hauteur $h$. Ainsi, pour un condensateur de hauteur $48 \mathrm{~mm}$, de capacité $76 \mu \mathrm{F}$, de résistance de couche $R_{\square}=8 \Omega$, de rayons interne et externe respectivement $4,5 \mathrm{~mm}$ et $32 \mathrm{~mm}$, les paramètres $\delta_{z}$ et $\alpha$, déterminés par l'équation (18), valent :

$$
\begin{aligned}
\delta_{z} & =17,7 \mathrm{~mm} \\
\alpha & =0,37 .
\end{aligned}
$$

Par ailleurs, pour les faibles valeurs de $\lambda$ (condensateur plat), le rapport entre $\delta_{z}$ et la hauteur $h$ du condensateur croît jusqu'à 0,6 .

3.2. Paramètres liÉs auX courants induits - Connaissant la valeur de $\delta_{z}$, nous pouvons définir l'impédance de surface $\underline{z}_{\mathrm{m}}$ de la métallisation entre les points A et $A$ ' ainsi que l'admittance surfacique $\underline{Y}_{\mathrm{sm}}$ de cette même métallisation entre les points A et B. Ces paramètres $\underline{z}_{\mathrm{m}}$ et $\underline{Y}_{\mathrm{sm}}$ sont analogues respectivement à l'impédance de surface du schoopage $\underline{z}_{\mathrm{s}}$ et à l'admittance surfacique du bobinage $\underline{Y}_{\mathrm{sc}}$.

Pour calculer le paramètre $\underline{Y}_{\mathrm{sm}}$, on peut supposer que le condensateur est constitué d'un empilement de couches métallisées de résistance de surface $R_{\square}$, espacées d'une largeur $a$, de hauteur $h_{0}$ et couvrant une surface $S=\ell \times L$ (Fig. 5).

Le nombre de couches métallisées considéré est $n=\frac{\ell}{a}$, la résistance d'une armature entre les plans 1 et 2 est $R=R_{\square} \frac{h_{0}}{L}$ La conductance des $n$ armatures en parallèle est donc

$$
\gamma_{\mathbf{n}}=n \frac{1}{R}=\frac{\ell L}{a h_{0} R_{\square}}=\frac{S}{a h_{0} R_{\square}} .
$$




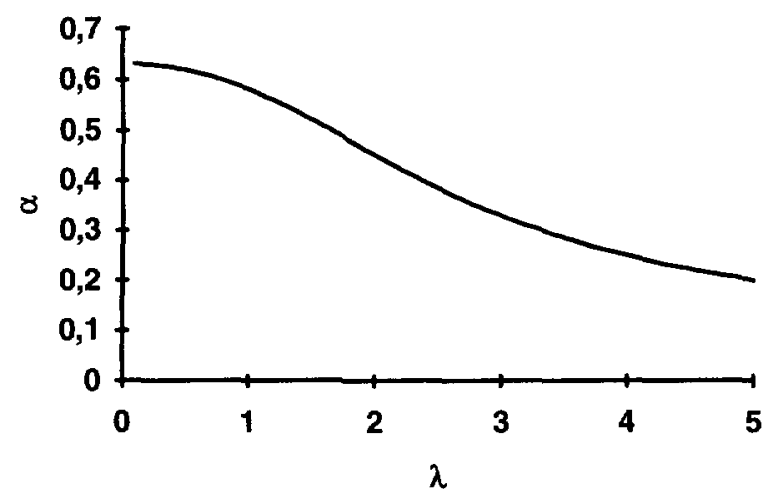

Fig. 4 - Évolution du paramètre $\alpha=\delta_{z} / h$ en fonction de $\lambda=2 h /\left(R_{\mathrm{nnt}}+R_{\mathrm{ext}}\right)$.

[Parameter $\alpha=\delta_{z} / h$ vs. $\lambda=2 h /\left(R_{\text {int }}+R_{\text {ext }}\right)$.]

L'admittance par unité de surface $\underline{Y}_{\mathrm{sm}}$ a, par conséquent, pour expression :

$$
\underline{Y}_{\mathrm{sm}}=\frac{\gamma_{n}}{S}=\frac{1}{a h_{0} R_{\square}}
$$

Le calcul du paramètre $\underline{z}_{\mathrm{m}}$ est plus délicat à mener car la moitié des armatures est courtcircuitée par le schoopage du haut et l'autre moitié par le schoopage du bas. On suppose que, pour les $n / 2$ armatures court-circuitées par le schoopage du bas, le courant $\underline{I}_{1 \mathrm{Am}}$ se répartit de façon uniforme dans une couche d'épaisseur $\delta_{z}$, de largeur $\ell$ et de longueur $x$ (Fig. 5). La résistance entre les plans 3 et 4 est alors $R_{\mathrm{b}}=2 \frac{a}{\ell} R_{\square} \frac{x}{\delta_{z}}$. Pour les $n / 2$ armatures court-circuitées par le schoopage du haut on trouve comme résistance $R_{h}=2 \frac{a}{\ell} R_{\square} \frac{x}{\delta_{z}}$. En conséquence, l'impédance de surface $\underline{z}_{\mathrm{m}}$, qui correspond à la mise en parallèle des résistances $R_{\mathrm{b}}$ et $R_{\mathrm{h}}$, vaut :

$$
\underline{z}_{\mathrm{m}}=\frac{a R_{\square}}{\delta_{z}}
$$

Les paramètres $\delta_{z}, h_{0}$ et $h_{\mathrm{m}}$ (Fig. $3 \mathrm{~b}$ ) sont par ailleurs liés :

$$
\begin{aligned}
h_{0} & =h-\delta_{z} \\
h_{\mathrm{m}} & =\frac{1}{2}\left(h_{0}^{\prime}+h\right)=h-\frac{\delta_{z}}{2} .
\end{aligned}
$$

Ainsi, pour la capacité de $76 \mu \mathrm{F}$ de l'exemple précédent, les différents paramètres qui caractérisent le condensateur valent :

$$
\begin{gathered}
\left.\begin{array}{c}
\delta_{z}=17,7 \mathrm{~mm} \\
\alpha=0,37
\end{array}\right\} \text { déterminés par l'équation (18) } \\
\left.\begin{array}{c}
h_{\mathrm{m}}=39,2 \mathrm{~mm} \\
h_{0}=30,3 \mathrm{~mm}
\end{array}\right\} \text { déterminés par l'équation }(22) \\
\underline{Y}_{\mathrm{sm}}=6,64 \times 10^{5} \mathrm{Sm}^{-2} \quad \text { déterminé par l'équation }(20) .
\end{gathered}
$$




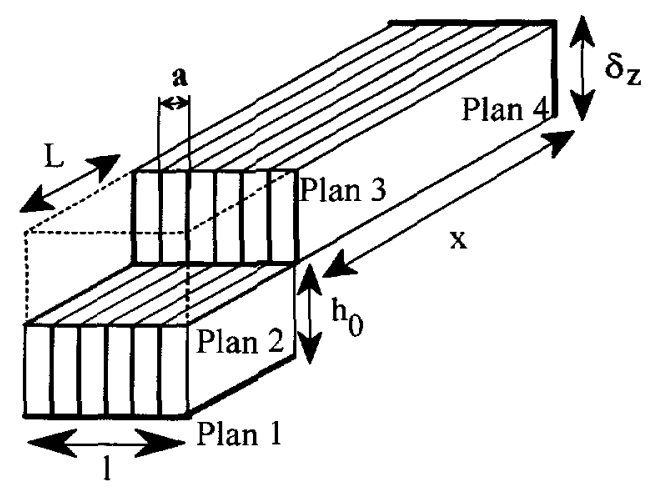

Fig. 5. - Structure des couches métalliques

[Structure of metallic layers]

Par analogie avec les équations (1) et (2), on prend la densité de courants induits dans les armatures suivant $\mathbf{e}_{z}$ égale à :

$$
\underline{J}_{\mathrm{sm}}=\left(\underline{V}_{\mathrm{m}}-j \omega A h_{\mathrm{m}}\right) \underline{Y}_{\mathrm{sm}}
$$

De même, par analogie avec l'équation (9), on définit les courants surfaciques $\underline{I}_{1 \mathrm{~m}}$ parallèles aux schoopages :

$$
\underline{\mathbf{I}}_{1 \mathrm{~m}}=-\frac{1}{z_{\mathrm{m}}} \frac{1}{r} \frac{\partial V_{\mathrm{m}}}{\partial \theta} \mathbf{e}_{\theta}
$$

Cette dernière équation ne fait intervenir que la direction $\mathbf{e}_{\theta}$ car les métallisations sont ellesmêmes parallèles à $\mathbf{e}_{\theta}$. Il n'existe donc pas de courants induits suivant $\mathbf{e}_{r}$.

\section{4. Équations de répartition des champs}

Les courants présents dans le bobinage du condensateur $\left(\underline{J}_{\mathrm{sc}}\right)$ et les schoopages $\left(\underline{\mathbf{I}}_{1}\right)$ ne sont pas indépendants. Ainsi, la somme des courants qui pénètrent dans l'élément de schoopage A (Fig. 2) doit être nulle. En conséquence :

$$
\underline{J}_{\mathrm{sc}}=\operatorname{div} \underline{\mathbf{I}}_{1 \mathrm{~A}}
$$

De même, la somme des courants qui pénètrent dans l'élément de schoopage B (Fig. 2) doit être nulle :

$$
\underline{J}_{\mathrm{sc}}=-\operatorname{div} \underline{\mathrm{I}}_{1 \mathrm{~B}}
$$

Soit, en posant $\underline{\mathbf{I}}_{1}=\underline{\mathbf{I}}_{1 \mathrm{~B}}-\underline{\mathbf{I}}_{1 \mathrm{~A}}$ :

$$
2 \underline{J}_{\mathrm{sc}}=-\operatorname{div} \underline{\mathbf{I}}_{1} \text {. }
$$

Par analogie avec l'équation précédente, on obtient l'équation de conservation du courant dans les métallisations :

$$
2 \underline{J}_{\mathrm{sm}}=-\frac{1}{r} \frac{\partial \underline{I}_{1 \mathrm{~m}}}{\partial \theta}
$$

Il est à noter que cette dernière équation ainsi que les équations (23) et (24) ne font pas intervenir les courants $\underline{J}_{\text {sc }}$ qui circulent pourtant dans les mêmes schoopages et les mêmes métallisations que le courant $\underline{J}_{\mathrm{sm}}$. Le fait que ces courants n'apparaissent pas vient de l'hypothèse sur l'absence de couplages entre les courants $\underline{J}_{\mathrm{sc}}$ et $\underline{J}_{\mathrm{sm}}$. 


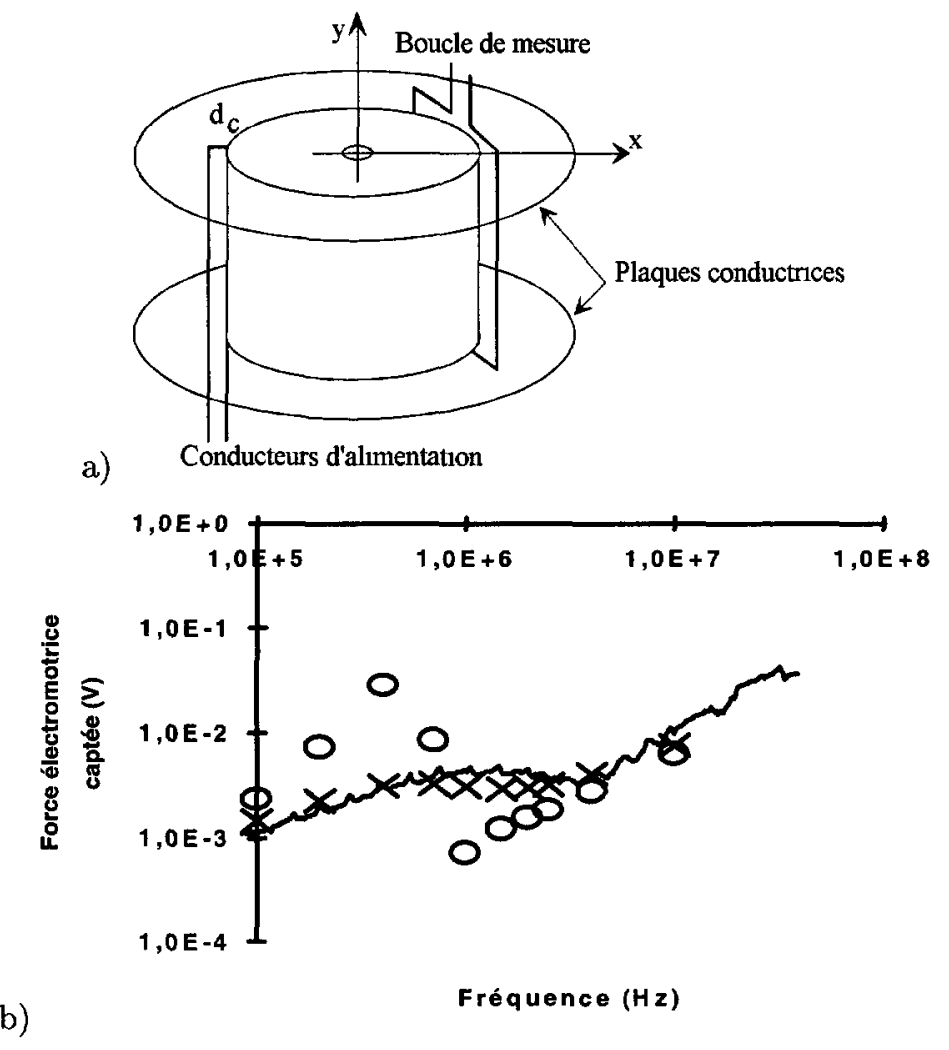

Fig. 6. - a) Représentation schématique du dispositif expérimental ; b) comparaison entre résultats expérimentaux (-) et théoriques pour $\delta_{z}=17,7 \mathrm{~mm}(\mathrm{x})$ et $\delta_{z}=0,1 \mathrm{~mm}(\mathrm{O})$.

[a) Schematic drawing of the experimental setup; b) comparıson between experımental (一) and theoretical results for $\delta_{z}=17.7 \mathrm{~mm}(x)$ and $\delta_{z}=0.1 \mathrm{~mm}(\mathrm{O})$.]

Enfin, la projection du potentiel vecteur magnétique $\mathbf{A}$ dépend de la densité des courants axiaux dans le condensateur :

$$
\frac{1}{\mu_{0}} \Delta \underline{A}=-\left(\underline{J}_{s c}+\underline{J}_{s m}\right)
$$

À partir des équations (1), (2), (9), (23), (24), (27), (28) et (29), on obtient un système d'équations différentielles qui lie $\underline{\mathbf{A}}, \underline{V}$ et $\underline{V}_{\mathrm{m}}$. Ce système a été résolu par la méthode des éléments finis en deux dimensions. Cette méthode ne permet certes pas d'atteindre une précision des résultats et une vitesse de calcul très élevées. Elle a cependant été utilisée afin de faciliter d'éventuelles modifications de la mise en équation.

Afin de déterminer la validité du modèle proposé, nous avons alimenté un condensateur de $76 \mu \mathrm{F}$ correspondant aux paramètres (19) par un conducteur externe asymétrique (Fig. 6a), éloigné $\mathrm{du}$ bord du bobinage d'une distance $d_{c}=4 \mathrm{~mm}$. La détermination directe des densités de courant dans le bobinage étant difficile à réaliser, nous avons choisi de mesurer l'induction magnétique à proximité du condensateur pour la comparer aux valeurs prévues par le modèle. Cette mesure a été réalisée à l'aide d'une spire placée à côté du bobinage pour capter la force électromotrice induite. L'influence de l'impédance des conducteurs sur les résultats expérimentaux est ainsi réduite. On peut noter, sur la figure $6 \mathrm{~b}$, la bonne concordance entre les résultats 


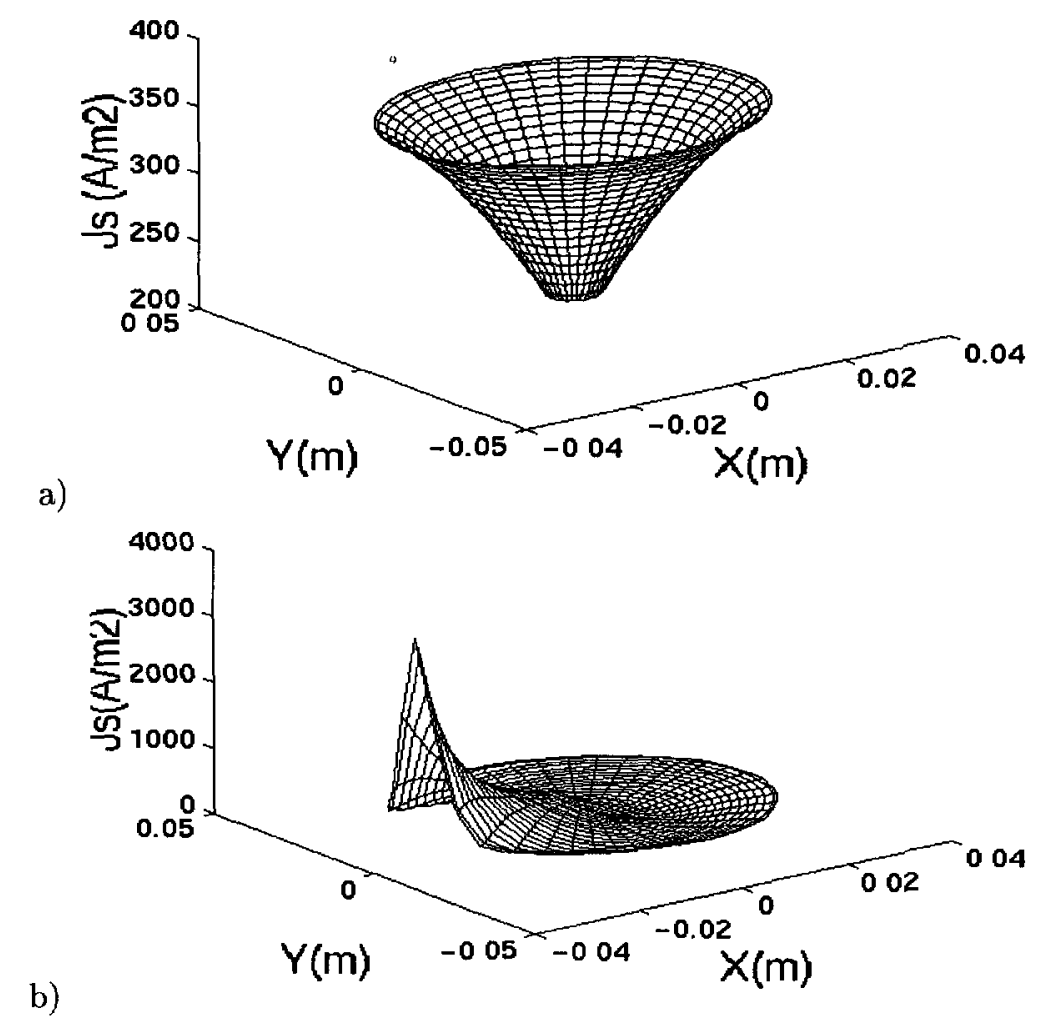

Fig. 7. - Densité de courant totale dans le condensateur $\underline{J}_{s}=\underline{J}_{\mathrm{sc}}+\underline{J}_{\mathrm{sm}}$. a) Conducteur interne centré ; b) conducteur externe décentré.]

[Total current density in capacitor $\underline{J}_{\mathrm{s}}=\underline{J}_{\mathrm{sc}}+\underline{J}_{\mathrm{sm}}$. a) Centered conductor; b) external conductor ]

expérimentaux (traits pleins) et les résultats théoriques obtenus pour $\delta_{z}=17,7 \mathrm{~mm}$ (croix). Par ailleurs, on a représenté les résultats de calculs effectués pour des courants de Foucault dans les métallisations réduits $\left(\delta_{z}=0,1 \mathrm{~mm}\right)$. On peut constater que, dans ce dernier cas, le tracé s'éloigne nettement du tracé expérimental. Ce résultat démontre l'importance des courants de Foucault dans les armatures et de la détermination correcte des paramètres $\delta_{z}$ et $\underline{z}_{\mathrm{m}}$.

La figure 7 donne les résultats de simulations pour deux dispositions du conducteur d'alimentation pour le condensateur de $76 \mu \mathrm{F}$ à $100 \mathrm{kHz}$. On constate que les courants se répartissent de façon relativement homogène quand le conducteur passe exactement au centre du condensateur (Fig. 7a). La densité maximale de courant est par ailleurs inférieure à $400 \mathrm{Am}^{-2}$. En revanche, quand le conducteur de retour du courant est placé à l'extérieur du condensateur (Fig. 7b), la densité surfacique de courant dans le condensateur devient fortement non homogène. Cette densité atteint une valeur proche de $4000 \mathrm{Am}^{-2}$ au voisinage du conducteur de retour. La différence entre les deux simulations provient essentiellement de la présence de forts courants induits dans le cas de répartitions non symétriques des champs et des courants.

La valeur élevée des courants dans les métallisations peut avoir comme conséquence une élévation de la température du condensateur préjudiciable à sa durée de vie. Il faut donc éviter de placer les conducteurs d'alimentation du condensateur de façon asymétrique pour éviter les échauffements supplémentaires. 


\section{Conclusion}

Les condensateurs bobinés à films secs métallisés sont constitués d'un nombre important de couches de diélectrique et de métallisation. Il est par conséquent très difficile de déterminer directement la répartition des champs électromagnétiques et des courants dans de tels condensateurs. Nous avons donc entrepris d'homogénéiser le matériau constitutif du bobinage, c'està-dire, à partir de la structure microscopique des films de diélectique et des métallisations, de déterminer les propriétés macroscopiques de ce bobinage.

En particulier, nous avons défini et calculé l'admittance surfacique du bobinage entre les deux schoopages ainsi que l'impédance de surface des schoopages. De façon similaire et pour prendre en compte les courants induits dans les métallisations, nous avons défini et calculé une admittance surfacique et une impédance de surface de métallisation.

Ces paramètres nous ont permis de déterminer la répartition des courants dans les condensateurs métallisés avec conducteur de courant décentré. Il a alors été possible de mettre en évidence l'importance des courants induits dans les métallisations et ainsi les risques d'échauffements importants liés à l'emploi d'un circuit non symétrique.

\section{Bibliographie}

[1] Sarjeant W.J., Capacitors, IEEE Trans. Elec. Insulation 25 (1990) 861-922.

[2] Mouriès G., Condensateurs utilisés en électronique de puissance, Tech. Ing. (1980) D-3-I, D 3280 .

[3] Matagne E., Contribution à la modélisation des dispositifs électrotechniques en vue de leur optimisation, Thèse de Doctorat (Louvain, 1991).

[4] Quivy D., Sur la permittivité et la perméabilité magnétique des mélanges, Bull. de la Soc. française des Électricıens, série 7, tome VIII, N88 (avril 1958).

[5] Périgord M., Contribution à l'étude des condensateurs utilisant des diélectriques solides en couches très minces, Thèse de docteur ès Sciences (Bordeaux, 1975).

[6] Joubert C., Béroual A. and Rojat G., Electric Field Distribution in All-Film Capacitors and Equivalent Circuit, J. of Appl. Phys. (à paraître).

[7] Ropa R. and Glaize C., Decrease of Inductance and Electromagnetic Interference in Power Electronics Capacitors, IEE Fith International Conference on Power Electronics and Variable-Speed Drives (Londres 26-28 octobre 1994) pp. 169-174.

[8] Joubert C., Rojat G. et Béroual A., Minimisation des inductances propres des condensateurs à film métallisé, J. Phys. III France 5 (1995) 1027-1038.

[9] Joubert C., Rojat G. et Béroual A., Perturbations électromagnétiques engendrées par les condensateurs à films métallisés, Congrès CEM 96 (Lille, 3-5 septembre 1996) pp. 432-436. 